\title{
Introduction to metabolomics and its applications in ophthalmology
}

\begin{abstract}
Metabolomics is the study of endogenous and exogenous metabolites in biological systems, which aims to provide comparative semi-quantitative information about all metabolites in the system. Metabolomics is an emerging and potentially powerful tool in ophthalmology research. It is therefore important for health professionals and researchers involved in the speciality to understand the basic principles of metabolomics experiments. This article provides an overview of the experimental workflow and examples of its use in ophthalmology research from the study of disease metabolism and pathogenesis to identification of biomarkers.

Eye (2016) 30, 773-783; doi:10.1038/eye.2016.37; published online 18 March 2016
\end{abstract}

\section{Introduction}

Recent genetic studies have made major contributions to understanding disease processes. However, biological processes operate through complex interactions between genes, RNA, proteins, and metabolites-the composite of this complex interaction network is defined as the interactome. ${ }^{1}$ Furthermore, environmental factors modify the interactome and consequently disease processes (Figure 1). These complex interactions define the phenotype of a disease. Hence, genetic research provides only part of the picture and ultimately understanding the interactome in disease pathogenesis provides the optimal route to developing new treatments and monitoring their effects. The desire to understand the interactome in disease processes has driven the development of new research methods. One important example is metabolomics, which is the study of endogenous and exogenous metabolites in biological systems. It aims to provide semi-quantitative information on metabolite abundances in a biological system.
SZ Tan ${ }^{1,2}$, P Begley, 3,4 , G Mullard ${ }^{3,4}$, KA Hollywood ${ }^{3,4,5}$ and PN Bishop $1,2,4$

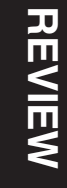

${ }^{1}$ Centre for Ophthalmology and Vision Sciences, Institute of Human Development,

Faculty of Medical and Human Sciences, University of Manchester, Manchester, UK of the phenotype of a biological system; genes determine what may happen and metabolites define what has happened. ${ }^{2}$

Metabolomics is used as a complementary approach to genomics, transcriptomics, and proteomics, and is the most recently developed technique; the first manuscript using the word metabolome was published in 1998. ${ }^{3}$ As the metabolome (defined as the complete set of metabolites found in a biological sample) is the end product of gene expression, it is thought to be a more sensitive method to measure the biological phenotype. ${ }^{4}$ Changes in the metabolome can occur rapidly in the timescale of seconds or minutes, hence it accurately reflects the state of disease at a given time point. ${ }^{5}$

Metabolomics has, in the past 10 years, become an increasingly attractive experimental method for studying disease pathogenesis, although it is still a relatively new method compared to its other-omic siblings. This review aims to provide a general overview of the metabolomics experimental workflow, and to equip readers with the basic knowledge required to understand and interpret results from metabolomics experiments. It will then provide examples of the use of metabolomics in eye research to date.

\section{Experimental workflow}

The typical workflow in metabolomic experiments has been described previously. ${ }^{1,6}$ The following paragraphs describe the basic steps involved in standard metabolomics experiments:

\section{Study strategies}

In general, two types of experimental workflow can be applied: untargeted studies and targeted studies.
${ }^{2}$ Department of

Ophthalmology, Manchester Royal Eye Hospital, Central Manchester University

Hospitals NHS Foundation Trust, Manchester Academic Health Sciences Centre, Manchester, UK

${ }^{3}$ Centre for Endocrinology and Diabetes, Institute of Human Development, Faculty of Medical and Human Sciences, University of Manchester, Manchester, UK

${ }^{4}$ Centre for Advanced Discovery and Experimental Therapeutics (CADET), Central Manchester University Hospitals NHS Foundation Trust, Manchester Academic Health Sciences Centre, Manchester, UK

${ }^{5}$ Faculty of Life Science, University of Manchester, Manchester, UK

Correspondence:

PN Bishop, Department of Ophthalmology, Manchester Royal Eye Hospital, Central Manchester University Hospitals NHS Foundation Trust, Manchester Academic Health Sciences Centre, Oxford Road, Manchester M13 9WL, UK Tel: +44 (0)161 275 5755; Fax: +44 (0)161 7010242 . E-mail: Paul.n.bishop@ manchester.ac.uk

Received: 1 September 2015 Accepted in revised form: 20 January 2016 Published online: 18 March 2016 


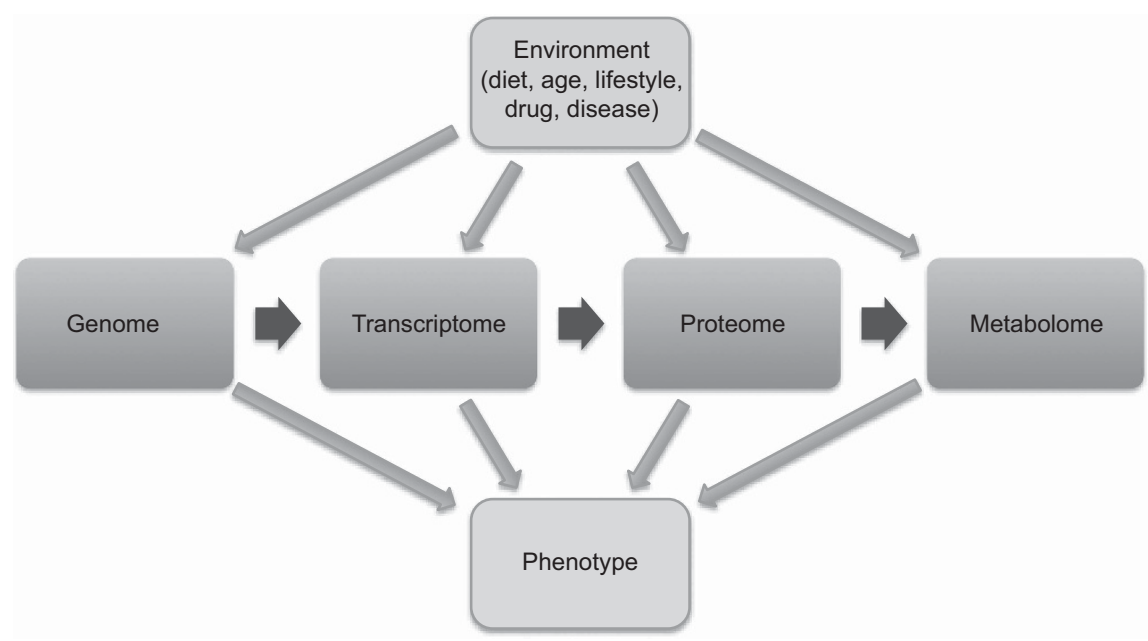

Figure 1 The interactome: the interaction between the genome, transcriptome, proteome, and metabolome with the environment in a biological system.

Untargeted studies, or metabolic profiling, are used to generate a hypotheses or when only a general hypothesis is available (ie, there are metabolic differences between cases and controls, but the specific differences are unknown). ${ }^{7}$ The experiment is designed to detect as many metabolites as possible, thereby attempting to span the entire metabolic network. Large-scale studies are usually required when metabolic profiling is applied clinically. Some authors recommend a three stage process for such studies; (a) discovery study, where numbers of subjects may be from 20 to 100 in each class sampled from two independent populations and this acts as a pilot study to identify specific groups of metabolites; (b) study validation, the study is repeated to validate previous findings from discovery study; and (c) cohort validation, where larger sample numbers in the region of 1000 s are employed. ${ }^{1}$

Targeted studies, on the other hand, focus on a small number of specific metabolites (typically fewer than 20, although broader targeted studies covering 100 or more metabolites are becoming more common) often in a similar metabolite class, having similar functions or involved in the same pathway. The method is hypothesis testing ${ }^{7}$ where metabolites of biological interest are already known. It provides high specificity, precision and accuracy. Targeted studies may be performed after metabolic profiling for result validation.

\section{Design of metabolomics experiments}

The two basic experimental designs used in metabolomics are (a) highly controlled laboratory studies in animal models or in vitro tissue culture and (b) clinical epidemiological studies to investigate pathogenesis of diseases, biomarkers, drug efficacy, and toxicity.
Laboratory studies tend to be small, as experimental, environmental, and genetic conditions can be highly controlled, allowing suitable statistical power to be achieved with a relatively low sample number. In clinical epidemiological studies, the metabolic status in the human population is highly diverse and can be affected by various factors such as diet, lifestyle, gender, medications, and age. Medium-to-large scaled studies are therefore required to detect differences within cohorts, and to reduce bias and false discovery rates. One strategy for decreasing the number of subjects is to use a longitudinal study design, where multiple time points are collected on individual subjects, and where possible, controlling the environment of the subjects. ${ }^{8}$

\section{Sample collection and preparation}

Turnover of metabolites over time (metabolic flux) can occur in seconds to minutes, unlike changes in levels of proteins and transcripts, which typically occur over minutes or hours. ${ }^{1}$ Therefore, sample collection and preparation are one of the most crucial steps in metabolomics experiments, as suboptimal handling can significantly reduce the accuracy and precision of the results.

Samples for analysis can be in the form of blood, serum, plasma, urine, cerebrospinal fluid (CSF), solid tissues, and cells. In the eye, commonly used tissues or fluids are cornea, lens, retina, vitreous, and aqueous. Typically, $>30 \mathrm{mg}$ wet tissue is recommended for untargeted studies and this can be challenging particularly in small animal models. Different sample types require different collection processes and preparation although the principles remain similar. The aims of this stage are to quench metabolic activity in the samples and then to 
isolate or extract the metabolites in an appropriate solvent for the analytical instrument. Quenching is performed to stop or slow down the metabolic activities so that metabolic flux is minimised or eliminated from the sample as soon as possible after collection. It can be performed by reducing the temperature to sub-zero immediately after collection and storing it at $-80^{\circ} \mathrm{C}$ until the sample is ready for further processing. ${ }^{9}$ Other methods used in quenching include increasing the temperature of the sample or introducing organic solvents with the aim of denaturing enzymes required for metabolism. ${ }^{1}$ The biological samples obtained are highly complex and requires 'extraction' to separate the metabolites of interest from a complex sample of matrix, which may include proteins, carbohydrates, DNA, and inorganic salts. Different extraction techniques are available and the process is dependent on the type of sample, experimental strategy (targeted vs metabolic profiling), and analytical instrument to be used. In general, targeted studies use specific analytical methods optimised for the metabolites of interest. By contrast, metabolic profiling is designed to detect a broad range of metabolites with different physicochemical properties in one sample and a standard analytical method, believed to provide the greatest metabolome coverage, is applied. Further details of extraction techniques are provided in a review by Dunn et al. ${ }^{1}$ Alternatively, intact tissue samples can be examined by MAS (magic angle spinning) nuclear magnetic resonance (NMR) spectroscopy (see below) avoiding possible negative effects of extraction on the composition of the samples.

\section{Analytical instruments}

The rapid development of metabolomics in recent times is mainly attributed to the advances in the instrumentation used to detect metabolites. One of the advantages of metabolomic experiments over, for example, proteomics is that higher sample throughput is possible. Depending on the type of sample, the analytical throughput can be tens of samples a day. ${ }^{2}$ Automation of sample preparation and loading also means that the instruments can operate for $24 \mathrm{~h}$ a day. Traditionally, the study of metabolism focused on single or specific metabolic pathways. However, with advances in instrument sensitivity, development in mathematical data transformation and powerful statistical methods, large numbers of endogenous metabolites can now be analysed in a single sample. ${ }^{8}$ The two most frequently used analytical techniques are (a) mass spectrometry (MS); and (b) NMR spectroscopy.

Mass spectrometry MS is an analytical technique that measures the mass of a molecule by measuring the mass- to-charge ratio $(\mathrm{m} / \mathrm{z})$ of its ions. Mass spectrometers operate in positive and negative ion modes (either as separate experiments or by polarity switching during analyses) and detect species that can be ionised by the addition or removal of a proton, or by the addition of another ionic species. The ion signal is processed into mass spectra, and the detected peaks can provide molecular weight and in some cases structural information. ${ }^{1}$ Separation techniques are often used before the compounds are introduced into the mass spectrometer to enhance sensitivity and aid the identification of metabolites; examples include gas chromatography mass spectrometry (GC-MS), liquid chromatography mass spectrometry (LC-MS), and capillary electrophoresis mass spectrometry (CE-MS) ${ }^{10}$ Each type of chromatography exploits the physicochemical properties of the metabolites in order to produce a separation of the mixture; volatility (vapour pressure) is used in gas chromatography, polarity (hydrophilicity/hydrophobicity) in liquid chromatography, and a combination of polarisibility and molecular shape in capillary electrophoresis. Chromatography works on the principle that various constituents travel at different speeds under set conditions and each constituent has a characteristic retention time, that is, the time it takes to pass from the inlet to the detector of the chromatography system. Figure 2 shows an exemplar $\mathrm{m} / \mathrm{z} 73$ single ion chromatogram of a human brain extract following GC-MS. Although GC-MS is largely limited to analysing low-molecular weight metabolites, LC-MS and CE-MS allow higher molecular weight metabolites to be analysed. This has broadened the applicability of MS in metabolomics. Details of how the instruments work can be found in other review papers. ${ }^{11-13}$

NMR spectroscopy NMR works on the basis that the magnetic properties of certain atomic nuclei can be used to determine the physical and chemical properties of atoms or molecules in which they are contained. The principle of NMR is that magnetic nuclei in a magnetic field absorb and re-emit electromagnetic radiation at a specific resonance frequency depending on the strength of the magnetic field and the magnetic properties of the isotope of the atom. ${ }^{1}$ Examples of commonly studied isotopes in NMR spectroscopy include ${ }^{1} \mathrm{H}$, carbon 13 $\left({ }^{13} \mathrm{C}\right),{ }^{19} \mathrm{~F}$, and phosphorus $31\left({ }^{31} \mathrm{P}\right)$. Fluid samples such as urine, serum, plasma, CSF, and so on, require minimal preparation for NMR-based experiments compared with MS experiments. High-resolution MAS NMR can also be used to analyse intact tissue samples in solid state. ${ }^{14,15}$ By spinning a sample at high frequency at the magic angle $\left(\theta_{\mathrm{m}}=54.74^{\circ}\right)$ with respect to the direction of the magnetic field, the normally broad spectrum becomes narrower, improving the resolution and hence analysis of the 


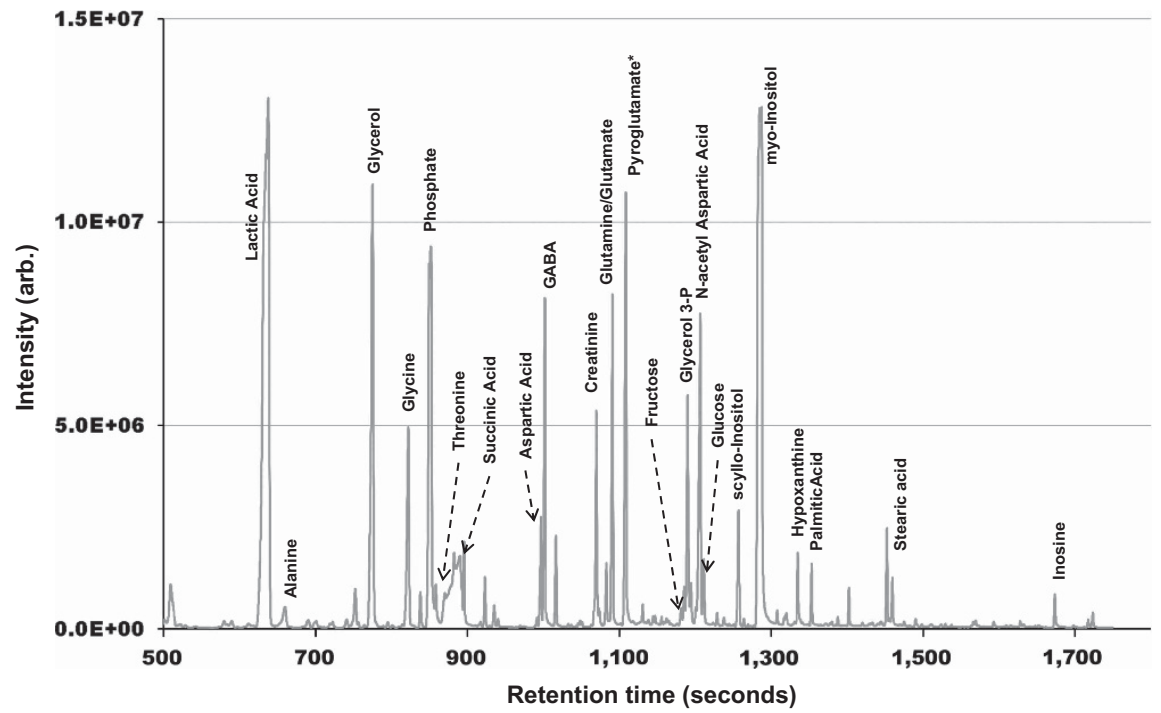

Figure 2 An exemplar $\mathrm{m} / \mathrm{z} 73$ single ion chromatogram of a human brain extract following GC-MS with some of the identified metabolites labelled; the $x$ axis represents retention time (defined as the characteristic time taken for a particular analyte to pass through the chromatography) and the $y$ axis represents relative signal intensity (Stefano Patassini, unpublished data, figure used with full permission).

spectrum. ${ }^{16}$ Recently, Kryczka et al ${ }^{17}$ applied MAS NMR to analyse human eye tissues for the first time and a total of 29 metabolites were identified from the cornea, iris, ciliary body, lens, and retina. The biochemical contents of the tissues were found to correspond to the biological functions.

Both MS and NMR can be used for targeted and untargeted metabolomics and are often complementary techniques. MS provides information on the molecular formula of a molecule, whereas NMR can differentiate between structural isomers. Compared with MS, NMR spectroscopy has the advantage of being highly quantitative, reproducible, and non-destructive to tissue samples, hence allowing samples to be analysed several times, but it is less sensitive than MS and generally requires larger sample volumes for analysis. On the other hand, MS is very sensitive and is able to detect a wide range of metabolites, hence it is usually the analytical instrument of choice in targeted studies in clinical chemistry laboratories. ${ }^{2}$ However, the extraction processes required prior to analysis can be relatively complex and time-consuming. ${ }^{18}$

\section{Data analysis}

One of the major challenges in metabolomics is the complexity and large volume of biochemical information obtained from either MS or NMR spectroscopy. Advances in statistical methods and modelling allow the rationalisation of the vast amount of data that can be generated so that important information can be extracted.
The raw data acquired from analytical instruments first needs to be converted into computer-readable formats compatible with relevant software packages. During the analysis of MS data, it undergoes a pre-processing step to provide alignment between samples and this usually converts continuous data to segmented data. In a chromatography-MS experiment, a 3D matrix of retention time vs mass vs intensity is converted to a $2 \mathrm{D}$ matrix of chromatographic peaks and peak areas or heights. This process is called 'deconvolution' and provides alignment of retention time and accurate mass. ${ }^{1}$ After the pre-processing step, the data are considered 'clean'. The next stage is normalisation of the data to remove concentration difference (so that high- and low-abundance features are given equal weighting in subsequent statistical analysis), scaling, outlier removal, and imputation of missing values. ${ }^{1}$

Univariate and multivariate statistical techniques can then be used to interpret the data. The most common goal of metabolomics experiments is to identify differences between experimental groups. With hundreds or thousands of metabolites in each class, it is impractical to visualise changes between groups by analysing metabolites individually; therefore, techniques are used to allow visualisation of global changes in the metabolome. One of the most widely applied methods is Principal Components Analysis (PCA). ${ }^{19}$ PCA allows data to be simplified without losing its main features. It provides at a glance information on multivariate variation among sample classes and it is usually performed at the initial stages of data analysis. PCA works by reducing the dimensionality of the data set, while retaining 


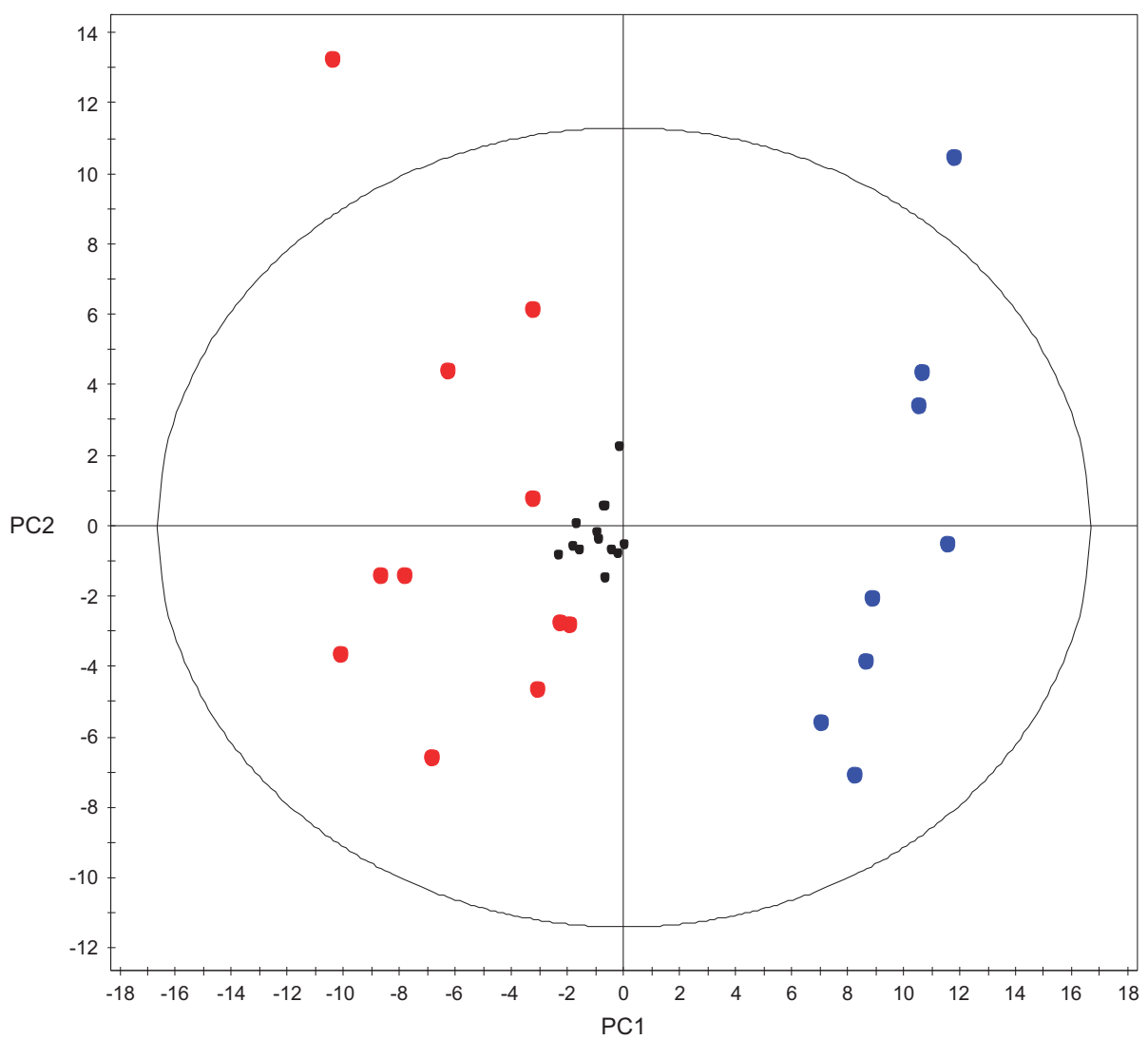

Figure 3 PCA plot (unpublished data, figure used with full permission) of control vs diabetic rat kidney in streptozotocin-induced diabetic rat model. The plot shows clear separation between the two groups indicating a substantial change in the metabolome. The biological difference between control and diabetic is large compared with the technical variation, and significant changes in individual metabolites (found later using univariate methods) are likely to reflect the disease state, rather than random variation. Black dots represent quality control; blue dots represent control kidney; red dots represent diabetic kidney.

characteristics contributing most to the variance. If the PCA plot shows separation between the study group and the controls, and the quality control samples are tightly grouped compared with the other classes in the experiment, the biological difference between the classes is significant compared with differences due to technical variation during analysis. Figure 3 shows an exemplar PCA. More details on PCA can be found in other reviews. ${ }^{1,20}$

Isolated metabolite markers can be analysed using univariate statistical tests such as the parametric Student's $t$-test, ANOVA, non-parametric Wilcoxon signed-rank test and Kruskal-Wallis test. ${ }^{1}$ A $P$-value of $<0.05$ is often used as the cutoff for statistical significance in biomedicine, but this is not suitable for metabolomics experiments where multiple tests are done simultaneously. More stringent univariate analyses (e.g., false discovery rate calculations or Bonferroni correction) are appropriate to provide validity to the analysis. ${ }^{8}$

\section{Metabolite identification}

The last step in the process is to assign a metabolite identity to the specific peaks generated from either MS or NMR. As discussed previously data sets generated from metabolic profiling experiments can be very large and the identification of metabolites on a large scale remains a bottleneck in metabolomics. ${ }^{21}$ Advances in technology have driven the development of comprehensive databases to tackle this problem.

Two classifications of identification are recognised in metabolomics: (1) Putative identification, where one or two molecular properties are used for identification but an authentic chemical standard is not used; (2) Definitive identification, which is the more accurate form of identification, employs at least two properties (typically the retention time and accurate mass and/or fragmentation mass spectrum and/or NMR spectrum) and compares these properties with an authentic chemical standard analysed under identical analytical conditions. Typically following putative identification, definitive 
identification is performed on selected metabolites using the relevant authentic standards. ${ }^{1}$

There are currently a number of different databases that are used in metabolomics. Some are mass-spectral-based databases for metabolite identification. There are also chemical structure databases, which can be searched by molecular mass. ${ }^{22}$ Different analytical instruments employ different databases although some of them overlap. Typically, spectra generated from the instruments are compared with reference compounds in a database and identities assigned. Among databases used in NMR spectroscopy are the Human Metabolome Database (HMDB, http://www.hmdb.ca), METLIN (http://metlin.scripps.edu), and Biological Magnetic Resonance Databank (http://www.bmrb.wisc.edu/ metabolomics/). Commonly used databases for GC-MS or LC-MS include US National Institute of Science and Technology database (NIST, http:/ / www.nist.gov/ srd/nist1a.htm), the Golm Metabolite Database (GMD, http:/ /csbdb.mpimp-golm.mpg.de/csbdb/gmd/gmd. html), MassBank (http://www.massbank.jp), METLIN, and the Madison Metabolomics Consortium Database (MMCD, http://mmcd.nmrfam.wisc.edu/). ${ }^{21}$ Although the metabolome databases are expanding every year, there are still significant numbers of unidentified metabolites in biological systems. Initiatives have been made towards creating a central reporting database to allow sharing of methodologies and results between laboratories. ${ }^{23,24}$ A detailed review of the database resources in metabolomics has recently been published. ${ }^{21}$

\section{Applications in ophthalmology}

The eye is believed to have its own distinct metabolome due to the blood-aqueous and blood-retinal barriers. The metabolome of aqueous and vitreous humour may be to a considerable extent represent local metabolism, with the systemic environment having a relatively minor effect. This theoretically makes eye an excellent organ for metabolomics experiments, although at present not much is known about the extent of inter-individual variations within the ocular environment. The following paragraphs describe how metabolomics has been used in ophthalmology for the characterisation of healthy biofluids, the study of tissue metabolism, the understanding of disease mechanisms, and the development of new therapies for ocular disease.

\section{Characterisation of healthy biofluids, cells, or tissues metabolome}

One application of metabolomics is to identify biomarkers or risk factors associated with diseases. The biomarkers can be clinically useful as diagnostic, screening, or prognostic tools. However, before disease biomarkers can be identified, studies to characterise the 'normal' metabolome are required and normal intra- and inter-individual variability needs to be determined.

${ }^{1} \mathrm{H}-\mathrm{NMR}$ has been used in a number of ocular studies. In one study of the cornea, 20-30 metabolites were detected and the presence of hypotaurine (precursor of taurine) and choline (precursor of acetylcholine) were established for the first time. ${ }^{25}$ Rabbit lens ${ }^{26}$ and animal vitreous ${ }^{27}$ have also been analysed using this technique.

LC-MS has the advantage of being more sensitive than ${ }^{1} \mathrm{H}-\mathrm{NMR}$ spectroscopy and is therefore able to identify higher number of metabolites in a given sample. Chen et $a l^{28}$ employed LC-MS in the characterisation of the human tear metabolome. Tears were collected from five healthy individuals using Schirmer strips and metabolites were extracted from these using a methanol/water solvent. Following LC-MS, 60 metabolites were detected, 44 of which had not previously been identified in tears. This analytical method may have utility in phenotyping ocular surface disease, as the tear composition may be reflective of ocular surface health. ${ }^{29}$

Different animal models are frequently used to investigate ocular tissue physiology and pathophysiology. The results from studies using different species are often extrapolated and used interchangeably with the assumption that they are biochemically comparable. However, Mains et al ${ }^{30}$ employed LC-MS to show that there were significant interspecies metabolic variations in the vitreous from healthy eyes highlighting that data derived from different animal models should be interpreted with care. The main metabolic differences between the species (sheep, pigs, and rabbits) suggested that there were differences in enzymatic activity across species and that diet may have a role in determining the metabolome of the vitreous.

\section{Study of tissue metabolism}

The study of metabolism is pertinent to the understanding of normal tissue function. NMR spectroscopy has been employed to study tissue metabolism since the early 1980s. Most studies were targeted studies looking at small groups of metabolites. Various types of NMR spectroscopy can be used to study different metabolic pathways. ${ }^{31} \mathrm{P}$ NMR spectroscopy has been used to investigate high-energy phosphate metabolites in extracts from the cornea and lens. The method was employed by Greiner et $a l^{31}$ to compare the metabolic status of fresh vs eye bank-stored cat corneas. It was found that the calculated corneal tissue-energy index (derived from the ratio of high-energy phosphate metabolites to low-energy phosphate metabolites) was significantly decreased in the eye bank-stored corneas, 
suggesting a reduced ability of these corneas to produce high-energy phosphate compounds crucial for cellular function, such as ATP. Lass et $a l^{32}$ went on to follow ATP changes in corneas stored in different media and investigated corneal metabolic status at given storage times. Using ${ }^{31} \mathrm{P}$ NMR spectroscopy, metabolic activity in the peripheral corneal epithelium was demonstrated to be higher than in the central area. ${ }^{33}$ This method had also been used to study the change in metabolic status in tissue at different ages and it was found that phosphatic metabolites changed significantly with age in corneas, indicating an overall decline in high-energy metabolism with ageing. ${ }^{34}$

${ }^{13} \mathrm{C}$ NMR spectroscopy was employed in studies to follow glucose and lactate formation in the rabbit ${ }^{35}$ and human post-mortem cornea. ${ }^{36}$ Deuterium (D or ${ }^{2} \mathrm{H}$ ) NMR spectroscopy has also been used to monitor glucose metabolism in rabbit corneas. ${ }^{37}$

Fris et $a l^{38}$ used ${ }^{1} \mathrm{H}-\mathrm{NMR}$ to investigate postnatal metabolic changes in rat lens and found a time-dependent increase in taurine, hypotaurine, and myo-inositol concentrations at the age of 30 days compared with birth, but a significant decrease in these energy metabolites and amino acids at the age of 60 days. Increased production of fructose and fructose-3-phophate was also demonstrated with age in rat lens. ${ }^{39} \mathrm{~A}$ recent study by Tsentalovich et $a l^{40}$ employing NMR and LC-MS showed a profound difference between the metabolome of normal and cataractous human lenses, where concentration of the most important metabolites such as anti-oxidants, UV filters, and osmolytes in the cataractous nucleus was 10 -fold lower than that in the normal nucleus. As the majority of these metabolites are synthesised in the lens epithelial layer, this provides evidence that age-related dysfunction of the epithelial layer may be responsible for the development of cataract.

\section{Study of disease pathology}

One of the earlier applications of metabolomics in ophthalmic conditions was in the evaluation of the effect of radiation on the eye. Animal studies using ${ }^{1} \mathrm{H}-\mathrm{NMR}$ demonstrated that UVB had a larger effect on the metabolic profile than UVA radiation. ${ }^{41}$ Changes in metabolites including a decrease in ascorbate, tyrosine, taurine/ hypotaurine, choline, myo-inositol, and an increase in glucose in the anterior segment of the eye in response to UVB light were detected. ${ }^{41,42}$ These metabolites included anti-oxidants (taurine/ hypotaurine, ${ }^{25,38,43,44}$ ascorbate, $^{45}$ and alanine ${ }^{46}$ ), a metabolite involved in growth and differentiation of lens epithelial cells (myo-inositol ${ }^{47,48}$ ), and a metabolite involved in the maintenance of cell membrane integrity (choline $\mathrm{e}^{47,49}$ ). Ascorbate is of particular importance in the cornea as it alone absorbs $77 \%$ of potentially damaging incident radiation at wavelengths $280-310 \mathrm{~nm} .{ }^{50}$ Treatment with dexamethasone resulted in a further depletion of corneal taurine and ascorbate and this could explain the accelerated process of cataract formation in patients on topical or systemic steroids. ${ }^{51}$

The biochemistry of contact lens-cornea interaction was investigated using ${ }^{31} \mathrm{P}$ NMR spectroscopy in the early years of metabolomics. It was found that polymethylmethacrylate lenses could cause reversible changes such as activation of anaerobic glycolysis and disturbance of membrane metabolite levels, whereas oxygen-permeable silicone lenses allowed maintenance of nearly normal metabolic patterns. ${ }^{52}$

Risa et $a l^{53}$ investigated changes in water-soluble metabolites in alkali-burned rabbit eyes using highresolution ${ }^{1} \mathrm{H}-\mathrm{NMR}$. The authors found that lactate was significantly increased not only in the cornea, but also in the lens. Glucose concentration was also increased in the cornea probably due to an influx from the aqueous humour following injury. Changes in metabolites including succinate, creatine, scyllo-myo-inositol, citrate, and ascorbate were also observed in the aqueous humour. A decrease in ascorbate concentration in the aqueous humour after alkali burn had been reported in several studies and treatment with exogenous ascorbate had been shown in animal studies to reduce the amount of corneal damage $e^{54,55}$ potentially by improving wound healing due to promotion of the secretion of mature collagen by corneal fibroblasts. ${ }^{56}$ These laboratory studies provided fundamental evidence of the benefit of vitamin C following chemical injury. Some clinicians now recommend high dose systemic vitamin $\mathrm{C}$ in the first few days following severe chemical injury.

A recent study by Karamichos et al ${ }^{57}$ looked at tear metabolite change in keratoconic patients without correction vs keratoconic patients who wore rigid gas-permeable contact lenses vs healthy subjects, employing LC-MS. The study found that metabolites involved in the urea cycle, citric acid cycle, and oxidative stress were up regulated in keratoconic patients compared with healthy subjects. Using LC-MS to investigate an in vitro model of keratoconus, increased levels of metabolites associated with oxidative stress were also observed, including lactate. ${ }^{29}$ Understanding the metabolic changes in keratoconus may help in the development of treatments for this condition.

Aqueous humour from a rat glaucoma model induced by intracameral sodium hyaluronate injection was analysed using NMR in a study by Mayodomo-Febrer et al. ${ }^{58}$ The study showed a reduced glucose level in the glaucomatous eyes compared with healthy eyes. The authors speculated that increased intraocular pressure could lead to a reduced energy supply to the anterior 
chamber tissue (reflected by the reduced glucose level), which in turns alters the outflow facility in the trabecular meshwork. Glutamate, a major neurotransmitter amino acid present in the retina was also found to be higher in the glaucoma model in this study. The toxic potential of glutamate to retinal ganglion cell (RGC) is well documented. ${ }^{59}$ Human $^{60}$ and animal studies ${ }^{59,61}$ have both identified elevated glutamate level in the vitreous of eyes with glaucomatous RGC death.

Several studies have used metabolomic techniques to study diabetic retinopathy. Santiago et al ${ }^{62}$ investigated metabolic changes in the retinas of streptozotocininduced diabetic rats using ${ }^{1} \mathrm{H}-\mathrm{NMR}$. The authors found that the levels of glucose were increased and lactate decreased in the diabetic rat retinas compared with age-matched controls. These findings were contrary to the general belief that an elevation of glucose concentration in the retina results in increased lactate concentration. Further data suggest that diabetes leads to decreased glycolysis in the retina.

\section{Biomarkers and risk factors for disease}

There have been studies aiming to identify clinically useful biomarkers. One of the most commonly used ocular tissues is vitreous due to its ease of access in human eyes during surgery and its anatomical proximity to the retina. Young et al ${ }^{63}$ employed ${ }^{1} \mathrm{H}-\mathrm{NMR}$ spectrometry to analyse a total of 42 vitreous samples obtained from patients undergoing pars plana vitrectomy for a variety of conditions including chronic non-infectious uveitis (CU), lens-induced uveitis (LIU), proliferative diabetic retinopathy (PDR), proliferative vitreoretinopathy (PVR), rhegmatogenous retinal detachment (RRD), candida endophthalmitis, and varicella zoster virus acute retinal necrosis. The authors undertook a PCA that showed clear separations between different clinical conditions and they were able to distinguish LIU from CU with a sensitivity of $78 \%$ and specificity of $85 \%$. In particular, they found that metabolites involved in the arginase pathway were significantly more abundant in LIU than CU, which can be explained by the different chronicity of the two conditions. The study showed for the first time that metabolomic analysis of vitreous humour samples might differentiate types of vitreoretinal diseases based on the different mix of metabolites present. Therefore, metabolic profiling of vitreous samples could be used in the future to guide diagnosis, prognosis, and measure response to treatment in various vitreoretinal diseases.

Barba et $a l^{64}$ applied ${ }^{1} \mathrm{H}-\mathrm{NMR}$ spectroscopy to explore the metabolic profile of vitreous humour in PDR. Vitreous samples were obtained during vitrectomy from 22 patients with type 1 diabetes mellitus with PDR and
22 non-diabetic patients with macular hole (control group). Among the metabolites present at higher levels in PDR vitreous samples compared with controls were lactate and glucose. This contrasts with the findings of Santiago et al, ${ }^{62}$ (see above) who demonstrated low lactate level in diabetic rat retina. These differences could reflect the different tissues that were analysed and/or leakage from the new vessels in PDR. Galactitol (a metabolite of galactose that is preferentially synthesised in the normoglycaemic state) and ascorbic acid (an anti-oxidant) were significantly lower in the samples from PDR patients compared with controls. PCA was able to clearly separate the samples into two classes. Li et a $6^{65}$ employed GC-MS to analyse the serum metabolome of diabetic patients and found a clear separation into three groups when serum samples from patients with pre-clinical, non-proliferative, and PDR were compared.

Li et $a l^{66}$ analysed the vitreous of patients with PVR and RRD using LC-MS. The authors identified markers associated with inflammation in the RRD cases and markers associated with proliferation in the PVR cases. Histidine metabolism and the citrate cycle were also found to be perturbed in both RRD and PVR.

Ugarte $e a^{6 l^{67}}$ observed multiple changes in the serum of streptozotocin-induced diabetic rats compared with non-diabetic controls including an elevation of 5-methoxytryptophan and a decrease in tryptophan serum concentrations in streptozotocin-induced diabetic rats. Similar changes have been found previously in the retina. ${ }^{68}$

More recently, metabolomics has been used to investigate the metabolic profiles in the plasma of patients with anterior uveitis ${ }^{69}$ and neovascular age-related macular degeneration (AMD) ${ }^{70}$ compared with controls employing LC-MS. Both studies demonstrated a distinct metabolic profile in the disease group. Pushpoth $e t a l^{71}$ performed metabolomics analysis on the serum and urine samples of patients with atrophic vs neovascular AMD and found clear separation between the two groups with some clustering of the neovascular and atrophic AMD samples, suggesting systemic metabolic perturbation in AMD with differences and similarities between the two forms of late AMD. However, it is important to point out that the studies described above are at an exploratory stage and none have undergone the validation required to definitively identify disease biomarkers.

Agudo-Barriuso et $^{772}$ studied changes in the rat retinal metabolome after optic nerve crush. A total of 27 metabolites were identified to discriminate between control retinas and 14 days post-optic nerve crush retinas, whereas 36 metabolites were identified to discriminate between retinas that were $24 \mathrm{~h}$ and 14 days post injury. They concluded that metabolic changes occur early in the retina following the crush injury and further changes 
occur later. These discriminating metabolites may be used as potential biomarkers for neuronal degeneration.

\section{Drug discovery, treatment evaluation, side effects, and toxicity}

Metabolomics enables a rapid and comprehensive assessment of how complex biological environments respond to drugs. It can be used in drug development to, for example, investigate mechanism of action or toxicity. Some examples are discussed below.

Song et $a l^{73}$ used ${ }^{1} \mathrm{H}-\mathrm{NMR}$ to evaluate the ocular hypotensive effects of a new drug, glycyrrhizin, in rabbits with glaucoma induced by intravitreal triamcinolone acetonide. Glycyrrhizin has been used to treat liver cirrhosis and is known to inhibit the formation of cortisol. The authors found that the intraocular pressure was significantly lower in rabbits treated with glycyrrhizin either pre- or post-triamcinolone injection compared with the no treatment group. ${ }^{1} \mathrm{H}-\mathrm{NMR}$ was subsequently used to study the effects of glucocorticoid and glycyrrhizin on ocular metabolism. It was found that triamcinolone affected carbohydrate metabolism via the tricarboxylic acid cycle, leading to a higher glucose level in the aqueous humour, and it was suggested that this could drive increased extracellular matrix deposition in the trabecular meshwork, causing increased resistance in aqueous outflow. However, glycyrrhizin partially inhibited the triamcinolone-induced effects on the tricarboxylic acid pathway. This study was limited by the number of rabbits used, but potentially sheds light on the pharmacodynamics of this potential new treatment and the mechanism of corticosteroid induced glaucoma.

Corneal collagen cross-linking is a relatively new treatment modality in corneal ectasias. Kryczka et $a l^{74}$ examined the influence of cross-linking on the metabolic profile of porcine corneas using (MAS)-NMR. The study found that the levels of formate, glutathione, and leucine, which contribute to the anti-oxidant defence system in the cross-linked corneas were significantly lower than in the controls in the early post-treatment phase. There was no major change in the metabolic profiles of the cross-linked corneas otherwise, suggesting that cornea cross-linking is a safe procedure. The use of anti-oxidants such as vitamin $\mathrm{C}$ to modulate wound healing after cross-linking can be considered to protect endothelial cells against apoptosis, especially in thin corneas.

\section{Conclusion}

The improving experimental techniques and instrumentation in the field of metabolomics has provided better understanding of the metabolism of normal ocular tissues and disease processes. However, these experimental approaches are still at its infancy and we have yet to witness the laboratory findings from modern metabolomics studies being translated into clinical practice. Although the eye is theoretically a good organ for metabolomics experiments due to its unique metabolome, ocular tissues or fluid are not always easily obtainable. One of the biggest pitfalls in metabolomics experiments is the large data set generated from the experiments and making biological sense of the metabolites is not always possible. Nevertheless, it is still an emerging and potentially powerful tool in ophthalmology research. Unique biological markers that can be identified through this technique will not only be valuable in aiding diagnosis in the future but will also serve as predictors of disease progression and treatment outcome in many ocular conditions. Clinicians should be aware of the basic principles of the field and interpret the experimental results with caution unless they have been fully and robustly validated.

\section{Conflict of interest}

The authors declare no conflict of interest.

\section{Acknowledgements}

Dr Shi Tan was an NIHR Academic clinical fellow.

\section{References}

1 Dunn WB, Broadhurst DI, Atherton HJ, Goodacre R, Griffin JL. Systems level studies of mammalian metabolomes: the roles of mass spectrometry and nuclear magnetic resonance spectroscopy. Chem Soc Rev 2011; 40(1): 387-426.

2 Dunn WB, Broadhurst D, Begley P, Zelena E, Francis-McIntyre $\mathrm{S}$, Anderson $\mathrm{N}$ et al. Procedures for large-scale metabolic profiling of serum and plasma using gas chromatography and liquid chromatography coupled to mass spectrometry. Nat Protoc 2011; 6(7): 1060-1083.

3 Oliver SG, Winson MK, Kell DB, Baganz F. Systematic functional analysis of the yeast genome. Trends Biotechnol 1998; 16(9): 373-378.

4 Ellis DI, Dunn WB, Griffin JL, Allwood JW, Goodacre R. Metabolic fingerprinting as a diagnostic tool. Pharmacogenomics 2007; 8(9): 1243-1266.

5 Goodacre R, Vaidyanathan S, Dunn WB, Harrigan GG, Kell DB. Metabolomics by numbers: acquiring and understanding global metabolite data. Trends Biotechnol 2004; 22(5): 245-252.

6 Brown M, Wedge DC, Goodacre R, Kell DB, Baker PN, Kenny LC et al. Automated workflows for accurate massbased putative metabolite identification in LC/MS-derived metabolomic datasets. Bioinformatics 2011; 27(8): 1108-1112.

7 Kell DB, Oliver SG. Here is the evidence, now what is the hypothesis? The complementary roles of inductive and hypothesis-driven science in the post-genomic era. Bioessays 2004; 26(1): 99-105. 
8 Broadhurst DI, Kell DB. Statistical strategies for avoiding false discoveries in metabolomics and related experiments. Metabolomics 2006; 2(4): 171-196.

9 Villas-Bôas SG, Roessner U, Hansen MAE, Smedsgaard J, Nielsen J (eds). Metabolome Analysis: An Introduction. Wiley Interscience John Wiley \& Sons, Inc: USA, 2007.

$10 \mathrm{Lu} \mathrm{W}$, Bennett BD, Rabinowitz JD. Analytical strategies for LC-MS-based targeted metabolomics. J Chromatogr B Analyt Technol Biomed Life Sci 2008; 871(2): 236-242.

11 Dettmer K, Aronov PA, Hammock BD. Mass spectrometrybased metabolomics. Mass Spectrom Rev 2007; 26(1): 51-78.

12 Villas-Bôas SG, Mas S, Akesson M, Smedsgaard J, Nielsen J. Mass spectrometry in metabolome analysis. Mass Spectrom Rev 2005; 24(5): 613-646.

13 Dunn WB. Current trends and future requirements for the mass spectrometric investigation of microbial, mammalian and plant metabolomes. Phys Biol 2008; 5(1): 011001.

14 Moestue S, Sitter B, Bathen TF, Tessem MB, Gribbestad IS. HR MAS MR spectroscopy in metabolic characterization of cancer. Curr Top Med Chem 2011; 11(1): 2-26.

15 Midelfart A. Metabonomics-a new approach in ophthalmology. Acta Ophthalmol 2009; 87(7): 697-703.

16 Alia A, Ganapathy S, de Groot HJ. Magic Angle Spinning (MAS) NMR: a new tool to study the spatial and electronic structure of photosynthetic complexes. Photosynth Res 2009; 102(2-3): 415-425.

17 Kryczka T, Wylęgała E, Dobrowolski D, Midelfart A. NMR spectroscopy of human eye tissues: a new insight into ocular biochemistry. Scientific World J 2014; 2014: 546192.

18 Johnson $\mathrm{CH}$, Gonzalez FJ. Challenges and opportunities of metabolomics. J Cell Physiol 2012; 227(8): 2975-2981.

19 H. H. Analysis of a complex of statistical variables into principal components. J Educ Psychol 1933; 24: 417-441.

20 Young SP, Wallace GR. Metabolomic analysis of human disease and its application to the eye. J Ocul Biol Dis Infor 2009; 2(4): 235-242.

21 Go EP. Database resources in metabolomics: an overview. J Neuroimmune Pharmacol 2010; 5(1): 18-30.

22 Brown M, Dunn WB, Dobson P, Patel Y, Winder CL, Francis-McIntyre $\mathrm{S}$ et al. Mass spectrometry tools and metabolite-specific databases for molecular identification in metabolomics. Analyst 2009; 134(7): 1322-1332.

23 Jenkins H, Hardy N, Beckmann M, Draper J, Smith AR, Taylor J et al. A proposed framework for the description of plant metabolomics experiments and their results. Nat Biotechnol 2004; 22(12): 1601-1606.

24 Bino RJ, Hall RD, Fiehn O, Kopka J, Saito K, Draper J et al. Potential of metabolomics as a functional genomics tool. Trends Plant Sci 2004; 9(9): 418-425.

25 Midelfart A, Dybdahl A, Gribbestad IS. Metabolic analysis of the rabbit cornea by proton nuclear magnetic resonance spectroscopy. Ophthalmic Res 1996; 28(5): 319-329.

26 Midelfart A, Dybdahl A, Gribbestad S. Detection of different metabolites in the rabbit lens by high resolution 1H NMR spectroscopy. Curr Eye Res 1996; 15(12): 1175-1181.

27 Locci E, Scano P, Rosa MF, Nioi M, Noto A, Atzori L et al. A metabolomic approach to animal vitreous humor topographical composition: a pilot study. PLoS One 2014; 9(5): e97773.

28 Chen L, Zhou L, Chan EC, Neo J, Beuerman RW. Characterization of the human tear metabolome by LC-MS/MS. J Proteome Res 2011; 10(10): 4876-4882.
29 Karamichos D, Hutcheon AE, Rich CB, Trinkaus-Randall V, Asara JM, Zieske JD. In vitro model suggests oxidative stress involved in keratoconus disease. Sci Rep 2014; 4: 4608.

30 Mains J, Tan LE, Zhang T, Young L, Shi R, Wilson C. Species variation in small molecule components of animal vitreous. Invest Ophthalmol Vis Sci 2012; 53(8): 4778-4786.

31 Greiner JV, Lass JH, Glonek T. Metabolic status of fresh v eye-bank-processed corneas. A phosphorus nuclear magnetic resonance study. Arch Ophthalmol 1984; 102(11): 1676-1677.

32 Lass JH, Greiner JV, Medcalf SK, Kralik MR, Meneses P, Glonek T. Effects of moist-chamber and McCarey-Kaufman medium storage on the metabolic status of the cornea: a 31P-magnetic resonance analysis. Ophthalmic Res 1988; 20(6): 368-375.

33 Hayashi K, Cheng HM, Iwasaki M, Hua X, Kenyon KR. Metabolic analysis of reepithelializing rabbit cornea using p-31 nuclear magnetic-resonance spectroscopy. Graefes Arch Clin Exp Ophthalmol 1990; 228(1): 73-77.

34 Lass JH, Greiner JV, Merchant TE, Glonek T. The effects of age on phosphatic metabolites of the human cornea. Cornea 1995; 14(1): 89-94.

35 Gottsch JD, Chen CH, Stark WJ, Maumenee AE. Corneal metabolism monitored with NMR spectroscopy. Trans Am Ophthalmol Soc 1986; 84: 183-191.

36 Gottsch JD, Chen CH, Aguayo JB, Cousins JP, Strahlman ER, Stark WJ. Glycolytic activity in the human cornea monitored with nuclear magnetic resonance spectroscopy. Arch Ophthalmol 1986; 104(6): 886-889.

37 Aguayo JB, McLennan IJ, Graham Jr C, Cheng HM. Dynamic monitoring of corneal carbohydrate metabolism using highresolution deuterium NMR spectroscopy. Exp Eye Res 1988; 47(2): 337-343.

38 Fris M, Midelfart A. Postnatal biochemical changes in rat lens: an important factor in cataract models. Curr Eye Res 2007; 32(2): 95-103.

39 Lal S, Szwergold BS, Taylor AH, Randall WC, Kappler F, Brown TR. Production of fructose and fructose-3-phosphate in maturing rat lenses. Invest Ophthalmol Vis Sci 1995; 36(5): 969-973.

40 Tsentalovich YP, Verkhovod TD, Yanshole VV, Kiryutin AS, Yanshole LV, Fursova AZh et al. Metabolomic composition of normal aged and cataractous human lenses. Exp Eye Res 2015; 134: 15-23.

41 Tessem MB, Bathen TF, Cejkova J, Midelfart A. Effect of UV-A and UV-B irradiation on the metabolic profile of aqueous humor in rabbits analyzed by $1 \mathrm{H}$ NMR spectroscopy. Invest Ophthalmol Vis Sci 2005; 46(3): 776-781.

42 Risa O, Saether O, Lofgren S, Soderberg PG, Krane J, Midelfart A. Metabolic changes in rat lens after in vivo exposure to ultraviolet irradiation: measurements by high resolution MAS 1H NMR spectroscopy. Invest Ophthalmol Vis Sci 2004; 45(6): 1916-1921.

43 Huxtable RJ. Taurine in the central nervous system and the mammalian actions of taurine. Prog Neurobiol 1989; 32(6): 471-533.

44 Shioda R, Reinach PS, Hisatsune T, Miyamoto Y. Osmosensitive taurine transporter expression and activity in human corneal epithelial cells. Invest Ophthalmol Vis Sci 2002; 43(9): 2916-2922.

45 Rose RC, Bode AM. Ocular ascorbate transport and metabolism. Comp Biochem Physiol A Comp Physiol 1991; 100(2): 273-285. 
46 van Heyningen R. What happens to the human lens in cataract. Sci Am 1975; 2336: 70-72; 77-81.

47 Podskochy A, Gan L, Fagerholm P. Apoptosis in UV-exposed rabbit corneas. Cornea 2000; 19(1): 99-103.

48 Hightower K, McCready J. Physiological effects of UVB irradiation on cultured rabbit lens. Invest Ophthalmol Vis Sci 1992; 33(5): 1783-1787.

49 Blankenberg FG, Katsikis PD, Storrs RW, Beaulieu C, Spielman D, Chen JY et al. Quantitative analysis of apoptotic cell death using proton nuclear magnetic resonance spectroscopy. Blood 1997; 89(10): 3778-3786.

50 Brubaker RF, Bourne WM, Bachman LA, McLaren JW. Ascorbic acid content of human corneal epithelium. Invest Ophthalmol Vis Sci 2000; 41(7): 1681-1683.

51 Saether O, Krane J, Risa O, Cejkova J, Midelfart A. High-resolution MAS 1H NMR spectroscopic analysis of rabbit cornea after treatment with dexamethasone and exposure to UV-B radiation. Curr Eye Res 2005; 30(12): 1041-1049.

52 Tsubota K, Kenyon KR, Cheng HM. Hard contact lens-induced metabolic changes in rabbit corneas. Exp Eye Res 1989; 49(5): 769-775.

53 Risa O, Saether O, Midelfart A, Krane J, Cejkova J. Analysis of immediate changes of water-soluble metabolites in alkali-burned rabbit cornea, aqueous humor and lens by high-resolution 1H-NMR spectroscopy. Graefes Arch Clin Exp Ophthalmol 2002; 240(1): 49-55.

54 Levinson RA, Paterson CA, Pfister RR. Ascorbic acid prevents corneal ulceration and perforation following experimental alkali burns. Invest Ophthalmol 1976; 15(12): 986-993.

55 Pfister RR, Hayes SA, Paterson CA. The influence of parenteral ascorbate on the strength of corneal wounds. Invest Ophthalmol Vis Sci 1981; 21(1 Pt 1): 80-86.

56 Pfister RR. Chemical injuries of the eye. Ophthalmology 1983; 90(10): 1246-1253.

57 Karamichos D, Zieske JD, Sejersen H, Sarker-Nag A, Asara JM, Hjortdal J. Tear metabolite changes in keratoconus. Exp Eye Res 2015; 132: 1-8.

58 Mayordomo-Febrer A, López-Murcia M, Morales-Tatay JM, Monleón-Salvado D, Pinazo-Durán MD. Metabolomics of the aqueous humor in the rat glaucoma model induced by a series of intracamerular sodium hyaluronate injection. Exp Eye Res 2015; 131: 84-92.

59 Dreyer EB, Zurakowski D, Schumer RA, Podos SM, Lipton SA. Elevated glutamate levels in the vitreous body of humans and monkeys with glaucoma. Arch Ophthalmol 1996; 114(3): 299-305.

60 Honkanen RA, Baruah S, Zimmerman MB, Khanna CL, Weaver YK, Narkiewicz J et al. Vitreous amino acid concentrations in patients with glaucoma undergoing vitrectomy. Arch Ophthalmol 2003; 121(2): 183-188.

61 Carter-Dawson L, Crawford ML, Harwerth RS, Smith EL, Feldman R, Shen FF et al. Vitreal glutamate concentration in monkeys with experimental glaucoma. Invest Ophthalmol Vis Sci 2002; 43(8): 2633-2637.

62 Santiago AR, Garrido MJ, Cristovao AJ, Duarte JM, Carvalho RA, Ambrosio AF. Evaluation of the impact of diabetes on retinal metabolites by NMR spectroscopy. Curr Eye Res 2010; 35: 992-1001.

63 Young SP, Nessim M, Falciani F, Trevino V, Banerjee SP, Scott RA et al. Metabolomic analysis of human vitreous humor differentiates ocular inflammatory disease. Mol Vis 2009; 15: 1210-1217.

64 Barba I, Garcia-Ramírez M, Hernández C, Alonso MA, Masmiquel L, García-Dorado D et al. Metabolic fingerprints of proliferative diabetic retinopathy: an 1H-NMR-based metabonomic approach using vitreous humor. Invest Ophthalmol Vis Sci 2010; 51(9): 4416-4421.

65 Li X, Luo X, Lu X, Duan J, Xu G. Metabolomics study of diabetic retinopathy using gas chromatography-mass spectrometry: a comparison of stages and subtypes diagnosed by Western and Chinese medicine. Mol Biosyst 2011; 7(7): 2228-2237.

66 Li M, Li H, Jiang P, Liu X, Xu D, Wang F. Investigating the pathological processes of rhegmatogenous retinal detachment and proliferative vitreoretinopathy with metabolomics analysis. Mol Biosyst 2014; 10(5): 1055-1062.

67 Ugarte M, Brown M, Hollywood KA, Cooper GJ, Bishop PN, Dunn WB. Metabolomic analysis of rat serum in streptozotocin-induced diabetes and after treatment with oral triethylenetetramine (TETA). Genome Med 2012; 4(4): 35.

68 Leino M, Airaksinen MM. Methoxyindoles of the retina. Med Biol 1985; 63(4): 160-169.

69 Guo J, Yan T, Bi H, Xie X, Wang X, Guo D et al. Plasma metabonomics study of the patients with acute anterior uveitis based on ultra-performance liquid chromatographymass spectrometry. Graefes Arch Clin Exp Ophthalmol 2014; 252(6): 925-934.

70 Osborn MP, Park Y, Parks MB, Burgess LG, Uppal K, Lee $\mathrm{K}$ et al. Metabolome-wide association study of neovascular age-related macular degeneration. PLoS One 2013; 8(8): e72737.

71 Pushpot S, Fitzpatrick M, Young S, Yang Y, Talks J, Wallace G. Metabolomic analysis in patients with age related macular degeneration. Invest Ophthalmol Vis Sci 2013; 54: (e-abstract 3662).

72 Agudo-Barriuso M, Lahoz A, Nadal-Nicolás FM, Sobrado-Calvo P, Piquer-Gil M, Díaz-Llopis M et al. Metabolomic changes in the rat retina after optic nerve crush. Invest Ophthalmol Vis Sci 2013; 54(6): 4249-4259.

73 Song Z, Gong Y, Liu H, Ren Q, Sun X. Glycyrrhizin could reduce ocular hypertension induced by triamcinolone acetonide in rabbits. Mol Vis 2011; 17(224): 2056-2064.

74 Kryczka T, Sel S, Wollensak G, Midelfart A. Metabolic profile of porcine corneas after photodynamic cross-linking treatment. Acta Ophthalmol 2012; 90(8): e658-e659. 